\title{
Measuring asthma control: a comparison of three classification systems
}

\author{
P.M. O’Byrne*,\#, H.K. Reddel ${ }^{\star}$, G. Eriksson ${ }^{+, \S}$, O. Östlund ${ }^{+}$, S. Peterson ${ }^{+}$, M.R. Sears ${ }^{\uparrow}$, \\ C. Jenkins $^{\tau}$, M. Humbert ${ }^{f}$, R. Buhl**, T.W. Harrison ${ }^{\# \#}$, S. Quirce ${ }^{\tau \uparrow}$ and E.D. Bateman ${ }^{++}$
}

ABSTRACT: There are various ways to classify asthma control; however, no classification is universally accepted. This retrospective analysis compared asthma control as assessed by the Asthma Control Questionnaire (5-item version; ACQ-5), Global Initiative for Asthma (GINA) or Gaining Optimal Asthma Control (GOAL) study criteria.

Pooled data at the final study week $(n=8,188)$ from three budesonide/formoterol maintenance and reliever therapy studies which measured ACQ-5 were stratified according to GINA or GOAL criteria and ACQ-5 score distribution. The percentages of patients with a controlled/partly controlled week (GINA), totally/well-controlled week (GOAL) and range of ACQ-5 cut-off points were compared.

Patients with GINA controlled, partly controlled and uncontrolled asthma had mean ACQ-5 scores of $0.43,0.75$ and 1.62 , respectively. Patients with GOAL totally controlled, well-controlled and uncontrolled asthma had ACQ-5 scores of $0.39,0.78$ and 1.63. The kappa measure of agreement was 0.80 for GINA and GOAL criteria, and 0.63 for GINA controlled/partly controlled and ACQ-5 $<1.00$. ACQ-5 detected clinically important improvements in $49 \%$ of patients who, according to GINA criteria, remained uncontrolled at the end of the study.

Asthma control measured by GINA or GOAL criteria provides similar results. GINA Controlled/ Partly Controlled and GOAL Totally Controlled/Well-Controlled correspond to ACQ-5 $<1.00$. The $A C Q-5$ is more responsive to change in a clinical trial setting than a categorical scale.

KEYWORDS: Asthma control, Asthma Control Questionnaire, Gaining Optimal Asthma Control, Global Initiative for Asthma

$\mathbf{A}$ chieving asthma control is the focus of all recently developed asthma treatment guidelines [1-3]. Overall asthma control consists of two domains. One is achieving day-today (or current) asthma control, indicated by the absence of asthma symptoms, minimal reliever use, normal activity levels and lung function values close to normal. The second domain is to minimise future risk to the patient by ensuring the absence of asthma exacerbations, the prevention of accelerated decline in lung function over time and no side-effects from medications.

The first study to use asthma control as the primary outcome was the Gaining Optimal Asthma Control (GOAL) study [4], which used a categorical scale to identify totally controlled or well-controlled asthma (table 1). The most widely disseminated asthma treatment guidelines, those of the Global Initiative for Asthma (GINA), use a slightly different scale to identify controlled, partly controlled or uncontrolled asthma [5] (table 2). These scales were developed based on expert opinion. A number of validated numerical scales have also been developed to quantify asthma control. These include the Asthma Control Questionnaire (ACQ) [6], the Asthma Control Test (ACT) [7] and the Asthma Therapy Assessment Questionnaire [8]. The most widely used of these assessment tools in clinical trials to date is the ACQ, which has been validated in three separate studies $[6,9,10]$ and for which the minimum clinically important difference has been established [6]. The ACQ was developed using expert opinion and originally contained seven items; however, a five-item version (ACQ-5) has been validated for use in clinical trials and epidemiological surveys [11]. Despite the fact that the GINA and GOAL categorical scales and the ACQ ordinal scale were all developed using expert clinical opinion, there is no reason to expect a close correlation between
AFFILIATIONS

*Michael G DeGroote School of

Medicine, Faculty of Health

Sciences, McMaster University, and

"Dept of Medicine, McMaster

University, Hamilton, ON, Canada.

"Clinical Management Group

Woolcock Institute of Medical

Research, Camperdown, Australia.

${ }^{+}$AstraZeneca Research and

Development, and

${ }^{5}$ Dept of Respiratory Medicine and

Allergology, University Hospital,

Lund, Sweden

fUniversité Paris-Sud 11, Service de

Pneumologie et Réanimation

Respiratoire, Hôpital Antoine Béclère, APHP, Clamart, France.

**Pulmonary Dept, Mainz University Hospital, Mainz, Germany.

\#\# Respiratory Biomedical Research Unit, City Hospital Campus, Nottingham University, Nottingham, UK.

"Dept of Allergy, Hospital La Paz, Comunidad Autónoma de Madrid,

Madrid, Spain.

${ }^{+}$Division of Pulmonology, Dept of Medicine, University of Cape Town, Cape Town, South Africa.

CORRESPONDENCE

P.M. O'Byrne, Michael G DeGroote School of Medicine, Faculty of Health Sciences, McMaster University,

Hamilton, ON, Canada

E-mail: obyrnep@mcmaster.ca

Received:

Aug 042009

Accepted after revision:

Jan 122010

First published online:

Jan 282010 
TABLE 1 Gaining Optimal Asthma Control (GOAL) definitions of asthma control

\begin{tabular}{|c|c|c|c|}
\hline Characteristic & Totally controlled ${ }^{\#}$ & Well-controlled" & Uncontrolled \\
\hline Daytime symptoms & None & $\leqslant 2$ days with symptom score $>1$ & Failure to fulfil totally controlled \\
\hline \multirow[t]{2}{*}{ Morning PEF } & $\geqslant 80 \%$ predicted every day & $\geqslant 80 \%$ predicted every day & \\
\hline & & All of the following characteristics: & \\
\hline Night-time awakening & None & None & \\
\hline Treatment-related AEs & None enforcing change in asthma therapy & None enforcing change in asthma therapy & \\
\hline
\end{tabular}

For the present analysis, GOAL criteria were evaluated over 1 week. PEF: peak expiratory flow; AE: adverse event. ${ }^{\#}$ : all of the following each week; ${ }^{\prime}: \geqslant 2$ of the following each week. Reproduced from [4] with permission from the publisher.

the two types of scale as the categorical scales are mainly based on symptom frequency whereas the ACQ is mainly based on symptom intensity or impact.

One study has reported a comparison of asthma control measured by the GOAL criteria with the ACQ [12]. This study identified that the crossover point between well-controlled and not well-controlled asthma is close to a score of 1.00 on the ACQ. However, to be confident that a patient has wellcontrolled asthma the ACQ score should be $\leqslant 0.75$, and to be confident that the patient has uncontrolled asthma the ACQ score should be $\geqslant 1.50$. Another study has evaluated the relationship between the GINA criteria and the ACT [13]; with the ACT, the lower the score the less well controlled the asthma. This study concluded that an ACT score of $\leqslant 19$ predicted partly controlled or uncontrolled asthma as defined by GINA. A further small study evaluated three different guideline-based tools (GINA, the National Asthma Education and Prevention Program and the Joint Task Force Practice Parameter) and compared them with the ACQ and ACT [14]. The authors concluded that there is good agreement between the guideline-based tools and moderate agreement between the ACT and ACQ, but poor agreement between the ACT or ACQ and the guideline-based tools [14]. There are no previous studies comparing GINA, GOAL and ACQ in a large population during treatment.
Three studies conducted to evaluate budesonide/formoterol (Symbicort ${ }^{\circledR}$ Turbuhaler; AstraZeneca, Lund, Sweden) as both maintenance and reliever therapy (Symbicort SMART ${ }_{\mathbb{B}}$; AstraZeneca) included measurements of ACQ-5, as well as diary recordings of symptoms, reliever use and lung function, which allowed a post hoc evaluation of asthma control using GINA or GOAL criteria. The purpose of this retrospective analysis is to: compare and contrast these different tools for evaluating asthma control in a large patient population; evaluate the agreement between ordinal and categorical scales for measuring asthma control; determine whether an ordinal scale such as the ACQ- 5 is more responsive to changes in asthma control in clinical trials than categorical scales used by GINA and GOAL.

\section{MATERIALS AND METHODS Measurement tools}

The asthma control measurement tools used for this analysis are those described by GINA [2], GOAL [4] (tables 1 and 2) and the ACQ-5 [9]. Both the GINA and GOAL evaluations were possible because patients recorded in daily diaries homemonitored peak expiratory flow (PEF), reliever use, asthma symptoms and nights with awakenings because of asthma symptoms. Patients completed the ACQ-5, consisting of five questions on symptom control; each of the questions was scored on a scale of $0-6$ where 0 represents excellent asthma

TABLE 2 Global Initiative for Asthma (GINA) definitions of asthma control

\begin{tabular}{|c|c|c|c|}
\hline Characteristic & Controlled $^{\#}$ & Partly controlled & Uncontrolled \\
\hline Daytime symptoms & None (twice or less/week) & More than twice per week & Three or more features \\
\hline Limitations of activities & None & Any & of "partly controlled" asthma \\
\hline Nocturnal symptoms/awakening & None & Any & present in any week \\
\hline Need for reliever/rescue treatment & None (twice or less per week) & More than twice per week & \\
\hline Lung function PEF or $\mathrm{FEV}_{1}{ }^{+}$ & Normal & $<80 \%$ predicted or personal best (if known) & \\
\hline Exacerbations $^{\S}$ & None & One or more per year ${ }^{f}$ & One in any week ${ }^{f . \# \#}$ \\
\hline
\end{tabular}

PEF: peak expiratory flow; FEV 1 : forced expiratory volume in $1 \mathrm{~s} .{ }^{*}$ : all of the following. ${ }^{\bullet}$ : any measure present in any week. ${ }^{+}$: not a reliable test for children aged $\leqslant 5$ yrs.

§. for the present analysis, GINA criteria were evaluated over 1 week. In this context, exacerbations occurring before the week of assessment are not included in the GINA assessment of asthma control. ${ }^{f}$ : any exacerbation should prompt review of maintenance treatment to ensure that it is adequate. ${ }^{\# \#}$ : an exacerbation in any week makes that an uncontrolled asthma week. 
control and 6 represents extremely poor control. The overall score from the ACQ-5 was the mean of the five responses. GINA, GOAL and ACQ-5 were assessed at baseline and for the final study week.

\section{Studies and population}

The data for the analysis were obtained from three doubleblind, randomised, parallel group studies (6-12 months in duration) which comprised all of the budesonide/formoterol maintenance and reliever therapy studies in which ACQ-5 was recorded (Table S1 in the supplementary material). The methodologies of the three studies have been published previously [15-17].

Two of the studies were of 6 months duration and compared budesonide/formoterol maintenance and reliever therapy with a higher maintenance dose of inhaled corticosteroid (ICS) in combination with a long-acting $\beta_{2}$-agonist (LABA) plus terbutaline (Bricanyl ${ }_{\ltimes} ;$ AstraZeneca) as needed $[15,16]$. The third was a 12-month study [17] which compared budesonide/formoterol maintenance and reliever therapy with the same maintenance

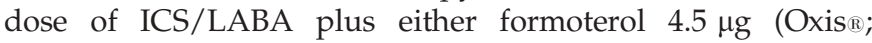
AstraZeneca) or terbutaline $0.4 \mathrm{mg}$ as needed for relief. All drugs were administered via Turbuhaler ${ }_{\circledast}$ (AstraZeneca) except for salmeterol/fluticasone (Seretide ${ }^{\mathrm{TM}}$; GlaxoSmithKline, Uxbridge, UK) which was delivered via either Diskus ${ }^{\mathrm{TM}}$ [15] or Evohaler ${ }^{\mathrm{TM}}$ (GlaxoSmithKline) [16].

Inclusion criteria for the three studies included age $\geqslant 12 \mathrm{yrs}$ and a diagnosis of asthma, a history of $\geqslant 1$ asthma exacerbation in the 12 months prior to study entry, use of ICS for at least 3 months prior to study entry, a forced expiratory volume in $1 \mathrm{~s}(\mathrm{FEV} 1) \geqslant 50 \%$ of predicted normal (pre-bronchodilator) with $\geqslant 12 \%$ reversibility following terbutaline $1.0 \mathrm{mg}$. Exclusion criteria included any respiratory infection affecting the patient's asthma or use of oral corticosteroids within 1 month of study entry. A total of 8,188 patients were available for inclusion in this retrospective analysis (table 3 ).

The studies were performed in accordance with the Declaration of Helsinki and Good Clinical Practice guidelines and were approved by independent ethics committees. Written informed consent was obtained from each adult patient; for under-age patients, informed consent from both the patient and their legal guardian was obtained.

\section{Statistical analyses}

Data from all three studies were pooled to evaluate asthma control as assessed by ACQ-5, GINA and GOAL criteria. GINA-based control (controlled or partly controlled) [2] and GOAL-based control (totally controlled or well-controlled) [4] were determined from the diary-card data and exacerbation data. For each patient, the day of the last on-treatment visit with recorded ACQ-5 scores was determined, and GINA and GOAL control criteria were determined from diary data in the week preceding the visit day. In order for a week to be counted as controlled (GINA) or totally controlled (GOAL), 7 days of diary data were required to have been recorded.

Pooled data were stratified according to GINA- and GOALbased criteria and for each control stratum the ACQ-5 score for the same week was described using mean, median, minimum, maximum and quartile values. The proportion of patients with a controlled or partly controlled week (GINA), a totally controlled or well-controlled week (GOAL) and an ACQ-5 score below the cut-off points $0.50,0.75,1.00,1.25,1.50$ and $\geqslant 1.50$ at the last week of study was also calculated. For each GINA control stratum the distribution of the ACQ-5 scores at the end of the study was plotted as the percentage of patients above each ACQ-5 score. Each of the ACQ cut-off points $0.50,0.75,1.00,1.25$ and 1.50 were evaluated as tests for GINA uncontrolled status in the end-of-treatment population in terms of sensitivity, specificity, positive and negative predictive values and the kappa measure of agreement. To assess the response to treatment, the same calculations were carried out for the last week in run-in. The proportion of patients that improved or deteriorated by $>0.50$ (minimal important difference) in ACQ-5 score during treatment was tabulated by GINA control status at the end of treatment. The number of patients satisfying each combination of GINA and GOAL criteria were tabulated in a $3 \times 3$ table and the kappa measure of agreement (where values $>0.8$ are considered indicative of "almost perfect agreement") [18, 19] was computed for GINA controlled compared with GOAL totally controlled asthma, for GINA uncontrolled compared with GOAL uncontrolled asthma, and as an overall measure for both comparisons.

Analyses were also conducted on the subpopulation of patients aged $<18$ yrs of age and on the impact of the morning PEF control criterion on GINA control versus ACQ-5. The proportion of patients classified into each GINA control class disregarding this criterion was also assessed (methodology and observations are included in the supplementary material).

\section{RESULTS}

\section{Studies and population}

Baseline characteristics were comparable between the three studies (table 3). At baseline, patients had a mean percentage predicted FEV1 of $72 \%$; all were using ICS (range of mean doses $709-761 \mu \mathrm{g} \cdot \mathrm{day}^{-1}$ ) and $46-58 \%$ of patients were also using a LABA. The median ACQ-5 score was 1.80 and $98 \%$ had uncontrolled asthma defined by GINA criteria.

\section{Comparison of GINA and GOAL criteria for asthma control}

In the last week of the studies the GINA and GOAL criteria identified a similar proportion of patients within each asthma control classification. Of the 1,476 patients considered controlled by the GINA criteria, 1,267 (86\%) were considered totally controlled and 209 (14\%) well-controlled by the GOAL criteria (table 4). Of the 1,291 patients considered totally controlled by GOAL criteria, all except 24 were considered controlled by GINA criteria. The kappa measure of agreement was 0.96. Similarly, of the 3,862 patients considered uncontrolled by the GINA criteria, 3,172 (82\%) were considered uncontrolled and 690 (18\%) well-controlled by the GOAL criteria (table 4) while, of the 3,690 patients considered uncontrolled by the GOAL criteria, 518 were considered partly controlled and no patients were considered Controlled by the GINA criteria. The kappa measure of agreement between the GINA uncontrolled classification and the GOAL uncontrolled classification was 0.80 . The overall kappa measure for all categories was 0.80 . 
TABLE 3 Baseline demographics of the three studies

BUD/FORM maintenance and reliever versus

Total of the three studies

\begin{tabular}{|c|c|c|c|}
\hline \multirow{2}{*}{$\begin{array}{l}\text { Same maintenance dose ICS/ } \\
\text { LABA + reliever } \\
\text { RABE [17] }\end{array}$} & \multicolumn{2}{|c|}{ Higher maintenance dose ICS/LABA + SABA } & \\
\hline & KUNA [16] & BOUSQUET [15] & \\
\hline 2856 & 3167 & 2165 & 8188 \\
\hline $1167(41)$ & $1337(42)$ & $824(38)$ & $3328(41)$ \\
\hline $42(12-89)$ & $38(11-83)$ & $40(12-80)$ & $40(11-89)$ \\
\hline $761(160-1600)$ & $744(100-3200)$ & $709(200-2000)^{\#}$ & $741(100-3200)^{\bullet}$ \\
\hline 58 & 46 & 55 & 53 \\
\hline $9(0-77)$ & $10(0-70)$ & $14(1-77)$ & $10(0-77)$ \\
\hline $72.0(39-101)$ & $72.9(29-143)$ & $70.6(45-222)$ & $72.0(29-222)$ \\
\hline $1.9(0.2-9.7)$ & $2.3(0.0-12.6)$ & $2.3(0.0-8.3)$ & $2.1(0.0-12.6)$ \\
\hline $10.8(0-100)$ & $8.7(0-100)$ & $10.8(0-100)$ & $10.0(0-100)$ \\
\hline $29.8(0-100)$ & $32.6(0-100)$ & $32.2(0-100)$ & $31.5(0-100)$ \\
\hline $1.8(0.0-5.4)$ & $2.0(0.0-5.6)$ & $1.8(0.0-5.4)$ & $1.8(0.0-5.6)$ \\
\hline 0 & 0 & $157(7)$ & $157(2)$ \\
\hline $2856(100)$ & $3167(100)$ & 2007 (93) & 8030 (98) \\
\hline
\end{tabular}

Data are presented as $\mathrm{n}(\%)$ or mean (range) unless stated otherwise. Data for duration of asthma are presented as medium (range). Demographic and baseline data for all patients with Asthma Control Questionnaire, 5-item version (ACQ-5) and Global Initiative for Asthma (GINA) control data at end of study and any diary data for the week preceding the last visit. BUD: budesonide; FORM: formoterol; ICS: inhaled corticosteroid; LABA: long-acting $\beta_{2}$-agonist; SABA: short-acting $\beta_{2}$-agonist; FEV1: forced expiratory volume in $1 \mathrm{~s}$; \% pred: \% predicted. ${ }^{*}: n=2,155 ; " n=8,178$.

\section{Relationship of GINA or GOAL criteria and ACQ-5 scores}

At the end of treatment, $53 \%$ of patients were controlled or partly controlled by GINA criteria and 55\% were totally controlled or well-controlled by GOAL criteria, while $50 \%$ of patients had an ACQ-5 score <1.0 (fig. 1). The GINA and GOAL control strata were similar in terms of ACQ-5 scores. Patients with controlled, partly controlled and uncontrolled asthma according to GINA criteria had mean (median) ACQ-5 scores of $0.43(0.20), 0.75(0.60)$ and $1.62(1.60)$, respectively (fig. 2), while patients with totally controlled, well-controlled and uncontrolled asthma according to GOAL criteria had mean (median) ACQ-5 scores of $0.39(0.20), 0.78(0.60)$ and 1.63 (1.60), respectively (fig 2).

\section{ACQ-5 cut-off points for uncontrolled asthma by GINA criteria}

When different ACQ-5 cut-off points were evaluated, 50.3\% of the GINA uncontrolled patients but $11.5 \%$ of the GINA controlled or partly controlled patients had ACQ-5 scores $\geqslant 1.50$ (fig. 3), translating into a specificity of $88.5 \%$ with a sensitivity of only $50.3 \%$ (table 5 ). An ACQ-5 score $\geqslant 0.75$ captured $80.3 \%$ of the GINA uncontrolled patients, making it a more sensitive test, but also included $37.1 \%$ of the controlled or partly controlled patients (fig. 3), thus reducing its specificity (table 5). The ACQ-5 cut-off point that provided the optimal balance between sensitivity and specificity for the GINA criteria was 1.0 (table 5). Of the 3,862 GINA uncontrolled

\begin{tabular}{|c|c|c|c|c|}
\hline \multirow[t]{2}{*}{ GINA criteria } & \multicolumn{3}{|c|}{ GOAL criteria } & \multirow[t]{2}{*}{ Total of the three studies } \\
\hline & Totally controlled & Well-controlled & Uncontrolled & \\
\hline Controlled & $1267(86)$ & $209(14)$ & 0 & $1476(18)^{\#}$ \\
\hline Total of the three studies & $1291(16)$ & 3207 (39) & $3690(45)$ & $8188(100)$ \\
\hline
\end{tabular}

Data are presented as $n(\%) .{ }^{*}: \%$ of all patients $(n=8,188)$ 


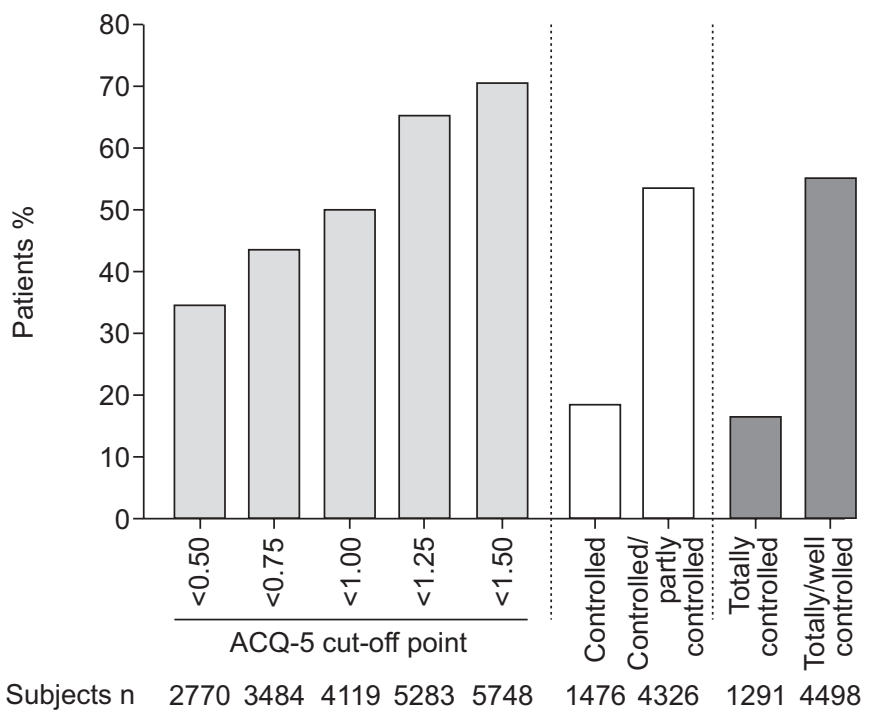

FIGURE 1. Proportion of patients achieving different levels of asthma control at end of treatment. Proportion of patients $(n=8,188)$ that satisfied different asthma control criteria in the final week of treatment (week preceding the last visit with recorded Asthma Control Questionnaire, 5-item version (ACQ-5) for each patient) pooled across all treatments. $\square$ : ACQ-5; $\square$ : Global Initiative for Asthma n; Gaining Optimal Asthma Control.

patients, $2,829(73.3 \%)$ satisfied ACQ-5 $\geqslant 1.00$ while, of the 4,069 patients with an ACQ-5 score $\geqslant 1.00$, the 2,829 uncontrolled patients comprised $69.5 \%$. Only 254 Controlled patients had an ACQ-5 score $\geqslant 1.00$. The kappa measure of agreement between ACQ- $5 \geqslant 1.00$ and the GINA uncontrolled classification was 0.63 (table 5). The sensitivity, specificity and

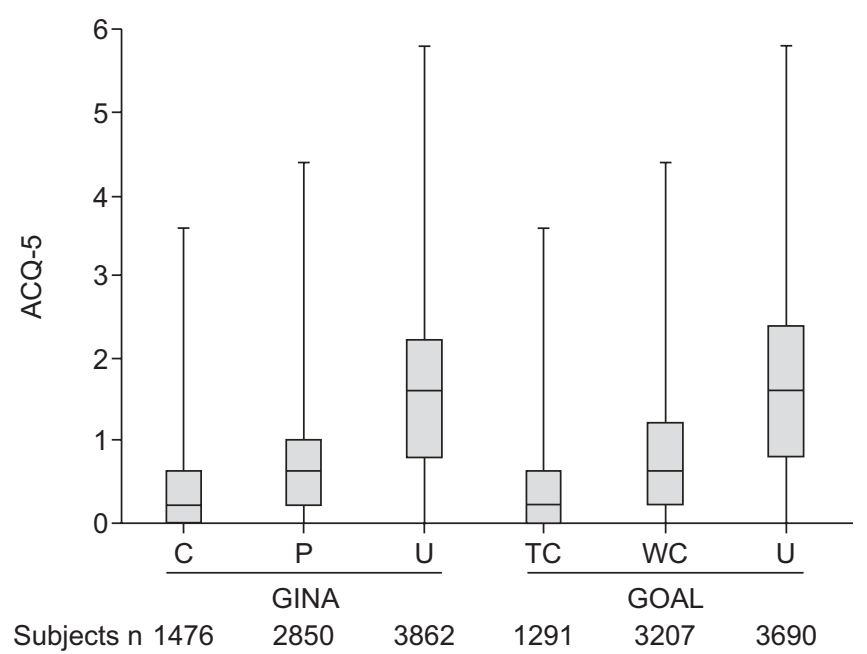

FIGURE 2. Asthma Control Questionnaire, 5-item version (ACQ-5) for different Global Initiative for Asthma (GINA) and Gaining Optimal Asthma Control (GOAL) levels of control at the end of treatment. Box-whisker plots showing total ACQ-5 score compared with GINA and GOAL defined asthma control at the week preceding the last visit with recorded ACQ-5 scores for each patient $(n=8,188)$. Boxes indicate the first and third quartile and median (-). Whiskers indicate the minimum and maximum $\mathrm{ACQ}-5$ values. C: controlled; $\mathrm{P}$ : partly controlled; U: uncontrolled; TC: totally controlled; WC: well-controlled.

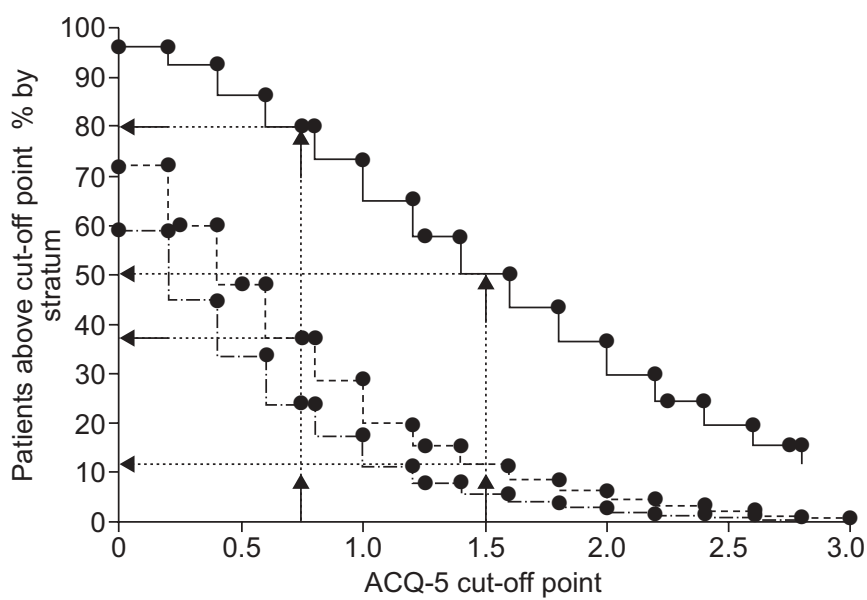

FIGURE 3. Percentage of patients with Asthma Control Questionnaire, 5-item version (ACQ-5) above each cut-off point by Global Initiative for Asthma (GINA) control stratum at last week of study. An ACQ- 5 score of $\geqslant 1.50$ was recorded by $50.3 \%$ of the GINA uncontrolled patients (-) but only $11.5 \%$ of the GINA controlled (-------) or partly controlled patients (- - - ) (see arrows). An ACQ-5 score of $\geqslant 0.75$ captured $80.3 \%$ of the GINA uncontrolled patients but also included $37.1 \%$ of the controlled or partly controlled patients (see arrows). ........ \% of uncontrolled and partly controlled patients with an ACQ-5 score $\geqslant 0.75$ and $\geqslant 1.50$, respectively.

positive and negative predictive values for a range of ACQ-5 scores are shown in table 5.

\section{Improvement during study as measured by GINA and ACQ-5}

At the end of treatment, $47 \%$ of patients remained uncontrolled by the GINA criteria (fig. 1). However, $49 \%$ of these uncontrolled patients showed an ACQ-5 improvement from baseline of at least 0.5 , which is considered clinically important (table 6) with the median ACQ-5 score decreasing from 2.2 at run-in to 1.6 at the end of study. For all patients, irrespective of control level, $61 \%$ showed a clinically important improvement in ACQ-5 score, with the median score decreasing from 1.8 to 0.8 (table 6).

\section{Relationship of the GINA or GOAL criteria and ACQ-5 scores in patients aged $<\mathbf{1 8}$ yrs}

As in the full population, similar proportions of patients aged $<18$ yrs $(n=1,217)$ were classified as controlled and totally controlled by GINA and GOAL criteria, respectively, or as partly controlled and well-controlled by GINA and GOAL criteria, respectively (fig S1 in the supplementary material). In children and adolescents, fewer patients were uncontrolled at end of study according to both GINA ( $41 \%$ versus $47 \%$ in the entire population) and GOAL criteria ( $39 \%$ versus $45 \%$ in the entire population). For ACQ-5, a larger fraction of patients were below each of the cut-off points 1.5, 1.25, 1.00, 0.75 and 0.50 compared with the entire population. Despite this, fewer patients were classified as controlled (14\% versus $18 \%)$ and totally controlled ( $13 \%$ versus $16 \%)$. The relationship between ACQ-5 cut-off points and the GINA categories is shown in table $\mathrm{S} 2$ in the supplementary material.

\section{DISCUSSION}

This post hoc analysis of data from $>8,100$ patients enrolled in the three budesonide/formoterol maintenance and reliever 


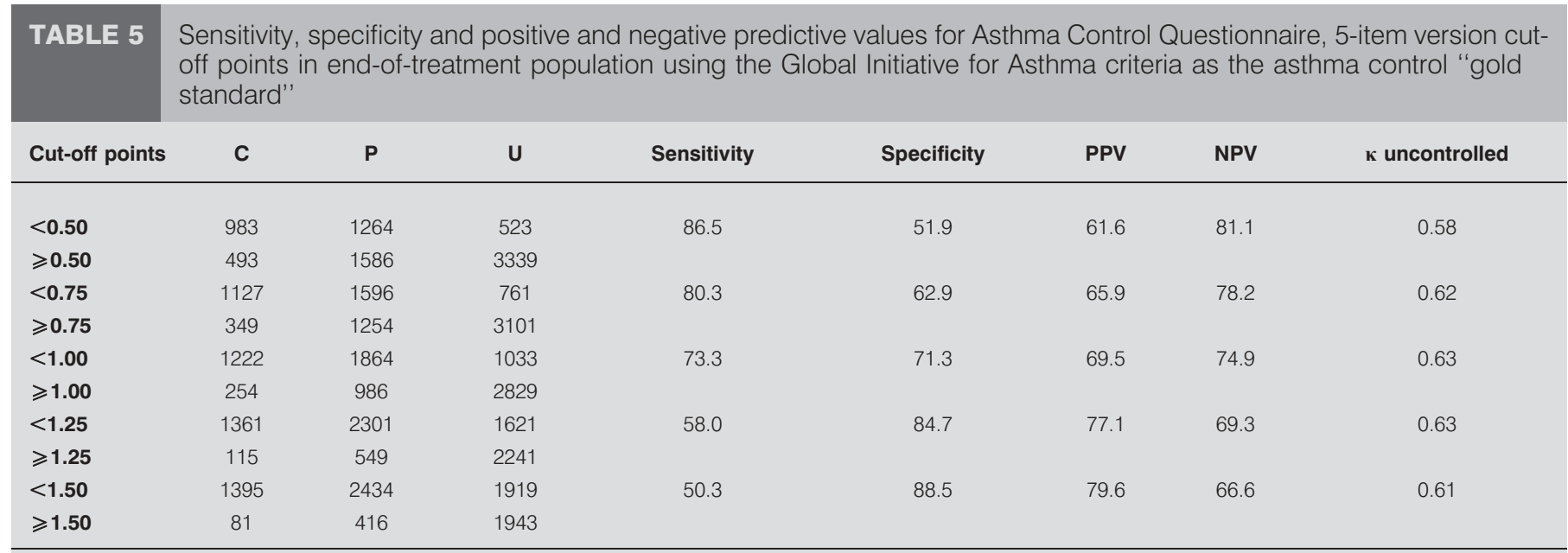

Data are presented as $\mathrm{n}$ or \%, unless otherwise stated. C: controlled; P: partly controlled; U: uncontrolled; PPV: positive predictive value; NPV: negative predictive value; $\kappa$ : kappa measure of agreement.

therapy studies in which the ACQ-5 score was measured compared asthma control as assessed by the ACQ-5, GINA or GOAL criteria. The analysis demonstrated the similarity between classification of asthma control as defined by GINA and GOAL. In addition, this analysis has shown that the percentages of patients considered by GINA criteria to have controlled and partly controlled asthma and by GOAL criteria to have totally controlled and well-controlled asthma are comparable to an ACQ-5 score of $<1.00$.

The similarity in the percentage of patients with different control classifications as measured by the GINA or GOAL criteria is not surprising as the criteria are reasonably similar. The differences that do exist are in the descriptions of controlled (GINA) and totally controlled (GOAL) asthma, where slightly more symptoms and rescue $\beta_{2}$-agonist use are permitted by GINA, and in the descriptions of the intermediate categories of partly controlled (GINA) and well-controlled (GOAL) asthma where, again, GINA is less stringent (table 2). These differences do not, however, result in any difference in which patients were considered to have controlled or totally controlled asthma, or partly controlled or well-controlled asthma, as reflected by a kappa value of 0.96 for GINA Controlled compared with GOAL totally controlled, and a kappa value of 0.80 for GINA uncontrolled compared with GOAL uncontrolled (kappa values exceeding 0.8 indicate almost perfect agreement [19]). These results are consistent with the findings of KHALILI et al. [14] who described good agreement between GINA and other guideline-based asthma control tools.

A previous study [12] that compared results obtained using the ACQ-5 and the evaluations of well-controlled and not wellcontrolled asthma from the GOAL study reported that the optimal cut-off point to be confident of well-controlled asthma was 0.75 (with a negative predictive value of 0.81 ) and to be confident of not well-controlled asthma was 1.50 (with a positive predictive value of 0.84 ). This means that, if a patient achieves an ACQ-5 score of 0.75 , there is only a $19 \%$ probability that asthma is not well-controlled. The current analysis extends these observations, using the GINA criteria for asthma control, and demonstrates that an ACQ- 5 cut-off point of 0.75 has a negative predictive value of 0.78 (giving a $22 \%$ probability that asthma is not controlled), while a cut-off point of 1.50 has a positive predictive value of 0.80 (giving a probability of $20 \%$ that asthma is controlled). However, when the same population was evaluated at study entry, which required asthma to be uncontrolled, the negative predictive value of a cut-off point of

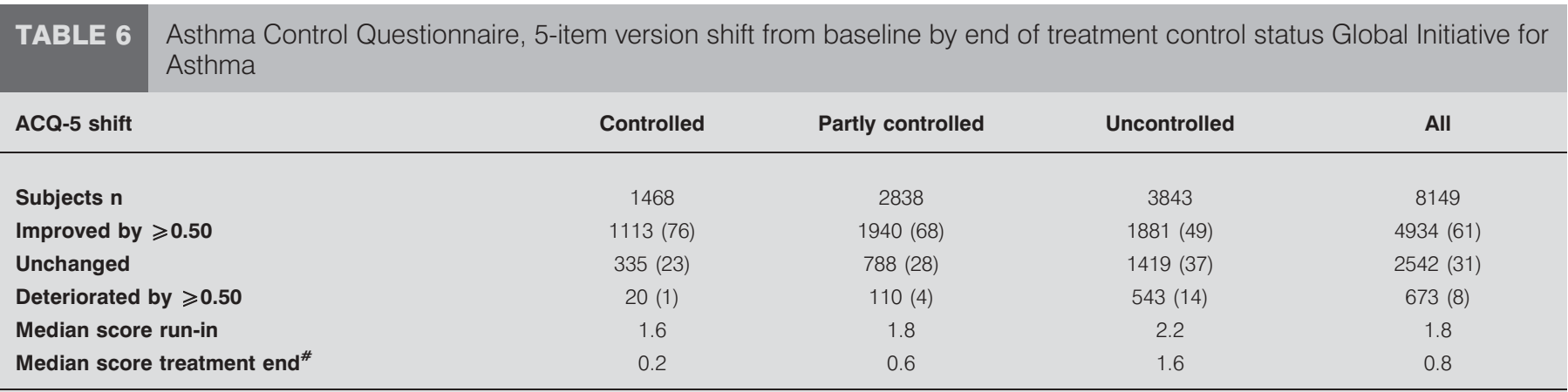

Data are presented as $n(\%)$, unless otherwise stated. * : controlled $n=1,476$; partly controlled $n=2,850$; uncontrolled $n=3,862$; all $n=8,188$. 
0.75 was 0.08 and the positive predictive value for a cut-off point of 1.50 was 0.99 (data not shown). This is because of the small number of patients at baseline who had an ACQ-5 score of $\leqslant 0.75$, making this a more homogeneous population than the population during the last week of treatment. This emphasises that ACQ-5 cut-off points must be based on an understanding of how representative the study population is of the population intended to take the test.

Another study has compared asthma control measured by the GINA criteria and the ACT in almost 3,000 asthmatic patients attending primary care [13]. The ACT was developed and validated to measure asthma control over the preceding 4 weeks [7]. In the comparison with the GINA criteria, an ACT score $\geqslant 20$ predicted GINA-defined controlled asthma $51 \%$ of the time, while a score of $\leqslant 19$ predicted partly controlled or uncontrolled asthma $94 \%$ of the time. However, the working definition of the GINA criteria differed from that used in the present analysis as, in the earlier analysis, exacerbations experienced during the previous 12 months were included in the assessment of partly controlled asthma; exclusion of this criterion substantially improved the positive predictive value of an ACT score of $\geqslant 20$. The ACT and ACQ have also been compared in a smaller population of patients, [20] and the authors concluded that these two measurement tools had comparable reliability, validity, accuracy and responsiveness.

The time course of change in ACQ- 5 in the present studies has recently been reported [21]. Overall, 61\% of patients improved by at least an ACQ- 5 score of 0.5 during the studies, which is considered to be a clinically important change [9]; however, in this group of asthma patients who at baseline were symptomatic on ICS or ICS/LABA therapy, $45-47 \%$ were still considered to have uncontrolled asthma by both the GINA and the GOAL criteria at the end of the studies, regardless of treatment allocation. This emphasises that even the use of effective treatment in controlled trials does not manage all patients with severe asthma optimally and that other treatment options may be needed for these patients. Nevertheless, with persistent therapy, even patients with uncontrolled asthma (49\%) showed improvement in ACQ-5 score by at least 0.5 units. These results suggest that an ordinal scale used by the ACQ is more responsive to changes in asthma control in a clinical trial setting, and is probably preferred in most asthma clinical trials where drugs are being evaluated or compared than a categorical scale.

The current analysis has limitations in that the GINA- and GOAL-defined asthma control categories were calculated retrospectively from study diary data; the ACQ-5 data were only analysed from the last week of the studies, and the patient population was required to have Uncontrolled asthma to enter into the studies and, therefore, is not necessarily representative of the asthma population as a whole.

The current results suggest that: measuring asthma control using the categorical scales used by GINA and GOAL provide comparable results, and an ACQ-5 score of $<1.00$ identifies a similar proportion of patients as the definition of controlled or partly controlled asthma described by GINA, and totally controlled or well-controlled asthma described by GOAL;
ACQ-5 cut-off points need to be based on an understanding of the study population intended to take the test; and in an asthma clinical trial setting, an ordinal scale to measure asthma control such as the ACQ-5 is more responsive to change than a categorical scale.

\section{SUPPORT STATEMENT}

This study was funded by AstraZeneca (Lund, Sweden).

\section{STATEMENT OF INTEREST}

Statements of interest for P.M. O'Byrne, G. Eriksson, O. Östlund, S. Peterson, M.R. Sears, C. Jenkins, R. Buhl, T.W. Harrison and E.D. Bateman, and for the study itself can be found at www.erj.ersjournals. $\mathrm{com} / \mathrm{misc} /$ statements.dtl

\section{ACKNOWLEDGEMENTS}

We would like to thank J. Sample (MediTech Media Ltd), who provided assistance in preparing the tables and figures and in formatting the manuscript for submission.

\section{REFERENCES}

1 National Asthma Education and Prevention Program, Expert Panel Report 3 (EPR-3): Guidelines for the diagnosis and management of asthma - Summary Report 2007. J Allergy Clin Immunol 2007; 120: Suppl. 5, S94-S138.

2 Bateman ED, Hurd SS, Barnes PJ, et al. Global strategy for asthma management and prevention: GINA executive summary. Eur Respir J 2008; 31: 143-178.

3 British Thoracic Society, Scottish Intercollegiate Guidelines Network. British Guideline on the Management of Asthma: A National Clinical Guideline. June 2009. www.sign.ac.uk/pdf/ sign101.pdf

4 Bateman ED, Boushey HA, Bousquet J, et al. Can guideline-defined asthma control be achieved? The Gaining Optimal Asthma ControL study. Am J Respir Crit Care Med 2004; 170: 836-844.

5 Global Initiative for Asthma. Global Strategy for Asthma Management and Prevention. Updated 2008. www.ginasthma. com/Guidelineitem.asp??11 $=2 \& 12=1 \&$ intId $=60$

6 Juniper EF, O'Byrne PM, Guyatt GH, et al. Development and validation of a questionnaire to measure asthma control. Eur Respir J 1999; 14: 902-907.

7 Nathan RA, Sorkness CA, Kosinski M, et al. Development of the asthma control test: a survey for assessing asthma control. J Allergy Clin Immunol 2004; 113: 59-65.

8 Skinner EA, Diette GB, Algatt-Bergstrom PJ, et al. The Asthma Therapy Assessment Questionnaire (ATAQ) for children and adolescents. Dis Manag 2004; 7: 305-313.

9 Juniper EF, Svensson K, Mörk AC, et al. Measurement properties and interpretation of three shortened versions of the asthma control questionnaire. Respir Med 2005; 99: 553-558.

10 Svensson K, Mork AC, Juniper EF. ACQ - is five out of seven items acceptable in large clinical studies? Qual Life Res 2003; 12: 771.

11 Juniper EF, O'Byrne PM, Roberts JN. Measuring asthma control in group studies: do we need airway calibre and rescue $\beta 2$-agonist use? Respir Med 2001; 95: 319-323.

12 Juniper EF, Bousquet J, Abetz L, et al. Identifying "wellcontrolled" and "not well-controlled" asthma using the Asthma Control Questionnaire. Respir Med 2006; 100: 616-621.

13 Thomas M, Kay S, Pike J, et al. The Asthma Control Test (ACT) as a predictor of GINA guideline-defined asthma control: analysis of a multinational cross-sectional survey. Prim Care Respir J 2009; 18: 41-49.

14 Khalili B, Boggs PB, Shi R, et al. Discrepancy between clinical asthma control assessment tools and fractional exhaled nitric oxide. Ann Allergy Asthma Immunol 2008; 101: 124-129. 
15 Bousquet J, Boulet LP, Peters MJ, et al. Budesonide/formoterol for maintenance and relief in uncontrolled asthma vs. high-dose salmeterol/fluticasone. Respir Med 2007; 101: 2437-2446.

16 Kuna P, Peters MJ, Manjra AI, et al. Effect of budesonide/ formoterol maintenance and reliever therapy on asthma exacerbations. Int J Clin Pract 2007; 61: 725-736.

17 Rabe KF, Atienza T, Magyar P, et al. Effect of budesonide in combination with formoterol for reliever therapy in asthma exacerbations: a randomised controlled, double-blind study. Lancet 2006; 368: 744-753.
18 Cohen J, ed. Statistical Power Analysis for the Behavioural Sciences. 2nd Edn. Hillsdale, Lawrence Erlbaum Associates, 1988

19 Landis JR, Koch GG. The measurement of observer agreement for categorical data. Biometrics 1977; 33: 159-174.

20 Wallenstein GV, Carranza-Rosenzweig J, Kosinski M, et al. A psychometric comparison of three patient-based measures of asthma control. Curr Med Res Opin 2007; 23: 369-377.

21 Bateman ED, Reddel HK, Ericksson G, et al. Overall asthma control: the relationship between current control and future risk. J Allergy Clin Immunol 2010; 125: 600-608. 